\title{
EL MODELO TRANSTEÓRICO COMO EXPERIENCIA COMUNITARIA EN LA SEPARACIÓN DE RESIDUOS SÓLIDOS DOMICILIARIOS PARA LA RECUPERACIÓN DE MATERIALES Y REDUCCIÓN DE LA CANTIDAD DE DESECHOS
}

\author{
MYRIAM CECILIA ESCOBAR RAMÍREZ \\ Candidata a Maestría en Enfermería. Universidad Cooperativa de Colombia, \\ Bucaramanga, Colombia. \\ miryam.escobar@campusucc.edu.co \\ NIDIA MARÍA TEJADA RIVERA \\ Especialista en Administración en Servicios de Salud. Universidad Cooperativa de Colombia, \\ Bucaramanga, Colombia. \\ nidia.tejada@campusucc.edu.co \\ MARÍA ENORIS ARANGO VASCO \\ Especialista en Gerencia en Servicios de Salud. Universidad Cooperativa de Colombia. \\ Bucaramanga, Colombia. \\ meavarango@yahoo.com.co
}

\section{Cómo citar este artículo:}

Escobar, M. \& Tejada, M.N. (2011). El Modelo Transteórico como experiencia comunitaria en la separación de residuos sólidos domiciliarios para la recuperación de materiales y reducción de la cantidad de desechos. Espiral, Revista de Docencia e Investigación. 1, (1), 83 - 92

\section{Resumen}

Los procesos de cambio son estrategias y técnicas cognitivas, afectivas y experienciales usadas para que las personas modifquen su comportamiento. En la perspectiva de intervenciones promotoras de la salud, son el eje de la facilitación y aceleración, representan principios básicos responsables de los cambios comportamentales esperados. En el Modelo Transteórico son el elemento fundamental para ser aplicado en el diseño de la estrategia: "Separe lo que bote antes de echarlo al pote", intervención promotora de la salud ambiental con impactos comportamentales directos e indirectos puesto que catalizan las transiciones de una etapa a otra, en la secuencia. El presente artículo da cuenta de la aplicación y sistematización de este modelo.

Palabras clave: Residuos sólidos, Modelo Transteórico, Educación Ambiental, reciclaje, cambios comportamentales.

\begin{abstract}
The processes of change are cognitive, affective and experiential strategies used for people to change their behavior. In the perspective of health-promoting interventions, these processes are the facilitating and accelerating core, which represent basic and responsible principles for expected behavioral changes. In the Transtheoretical Model, these processes are the key to be applied in the design of the strategy "Separate what you throw before throwing it to the trash", promoting intervention of environmental health with direct or indirect behavioral impacts that catalyze transitions from one stage to another in the sequence.
\end{abstract}

Key words: Solid waste, Transtheoretical Model, environmental education, recycling, behavioral changes. 


\section{Introducción}

La problemática ambiental mundial ocasionada por el incremento de los residuos sólidos se debe, en parte, a la falta de educación y responsabilidad ambiental para separarlos en la fuente y aprovecharlos nuevamente como materia prima para la fabricación de nuevos productos y Bucaramanga, capital del departamento de Santander, situado al Nor-oriente colombiano, no es la excepción. La ciudad santandereana no ha escuchado a Kant cuando afrma que todo ser organizado, debe organizarse él mismo. Así, la cantidad de residuos sólidos generados por la población y nueve municipios más de Santander alcanza diariamente las 750 toneladas y la disposición fnal es el relleno sanitario El Carrasco, según reporta la Empresa de Aseo de Bucaramanga (EMAB, 2010).

Este sitio es el destino fnal de materiales orgánicos e inorgánicos depositados en forma indiscriminada, todo ello causado por la cultura del consumismo y una errónea concepción de naturaleza que desconoce a Marx cuando sentencia: "el hombre vive de la naturaleza, quiere decir que la naturaleza es su cuerpo, con el que debe mantenerse unido para no morir". No es extraño, en consecuencia, que se imponga como norma de conducta interiorizada sin escrúpulo alguno, el inadecuado manejo de los residuos sólidos.

A lo anterior debemos sumar el crecimiento desaforado de la ciudad, la concentración de la población en la meseta, la existencia de asentamientos humanos subnormales en zonas de alto riesgo o de protección forestal y la existencia de una industria que no cuenta con el uso de tecnologías limpias para mitigar la degradación ambiental. (Plan Territorial de Salud, 2008)
Si se lograra aplicar la regla de las tres $\mathrm{R}$, REDUCIR, RECICLAR y REUTILIZAR, se obtendrían los materiales para adecuar un efciente sistema de relleno sanitario, con su consecuente benefcio en términos sociales, ambientales y económicos. De esta manera se realizaría el procesamiento de materiales orgánicos para producción de abonos completos y un aumento progresivo de los materiales inorgánicos que se pueden incorporar a procesos productivos, (Alcaldía de Pereira, 2008) Pero la complejidad del problema requiere la participación tanto de profesionales de la salud, como de las entidades comprometidas y de la comunidad en general para una solución defnitiva.
La Facultad de Salud, a través del Programa de Enfermería de la Universidad Cooperativa de Co- lombia, ejerce una infuencia de- terminante en el área de la salud pública y en el abordaje del pro- blema de los residuos sólidos tanto por su accesibilidad como líder de los centros de salud, como por su papel como educadores de salud

Frente a la gran problemática de concentración de residuos tanto industriales como en los hogares y la defciente utilización de los rellenos sanitarios, el gobierno colombiano ha implementado programas ambientales que se han incluido en la Legislación Ambiental Colombiana y son afnes a toda la normatividad que se ha creado para el cuidado del medio ambiente. En cuanto al tema del reciclaje, la reglamentación y parámetros que se ha creado para la recuperación de residuos sólidos se ha realizado por medio de planes guía como:

Plan de gestión de residuos sólidos (PGIRS); Plan para el Manejo Integral de Residuos Sólidos (PMIRS); Estrategia para la Estructuración del Sistema Organizado de Reciclaje, (SOR). 
Desde la Atención Primaria con la Promoción de la Salud el profesional de enfermería, cuenta con el modelo Transteórico de Prochaska y Di Clemente (1997), reconocido como un recurso innovador en el área de educación y promoción de la salud y considerado por diversos autores como un importante factor en la redefnición de la base teórica de las intervenciones del área en los últimos años (Ministerio del Medio Ambiente y Salud, 2003) Este modelo explica cómo la motivación cambia a lo largo del tiempo y cómo esto es importante para evaluar la disposición para el cambio. Este modelo del cambio del comportamiento en salud se consolidó durante los años noventa como una de las propuestas más innovadoras en el área de promoción de la salud y prevención de la enfermedad, por las posibilidades que ofrece para planear y ejecutar intervenciones a partir de las características específcas de las poblaciones o grupos a quienes están dirigidas las acciones. (Prochaska y Di Clemente, 1997:38-48)

Fundamentado en la premisa básica que, el cambio de comportamiento es un proceso y que las personas tienen diversos niveles de motivación, de intención de cambio, si bien, como afrma Maya (2001:14): “El hombre es responsable de su acción y ningún dios o demonio puede reemplazar dicha responsabilidad". Heráclito lo expresó con una frase contundente: "El comportamiento es el demonio del hombre", a pesar de ello, es posible planear intervenciones, programas, estrategias

"El hombre es responsable de su acción y ningún dios o demonio puede reemplazar dicha responsabilidad" que responden a las necesidades propias de las personas dentro de su grupo social o contexto natural comunitario $u$ organizacional. El modelo se apoya en una serie de postulados sobre la naturaleza del cambio de comportamiento y de las características de las intervenciones que pueden facilitar dicho cambio. (Prochaska \& DiClemente, 1997)

El tiempo en el Modelo Transteórico es muy importante para las etapas del cambio y para lograr integrar los procesos y principios explicativos del cambio comportamental.

Las etapas mediante las cuales el modelo transteórico explica la progresión del cambio son: Pre-contemplación, contemplación decisión, acción, mantenimiento y regresión. (Cabrera, 2000)

La estrategia "Separe lo que bote antes de echarlo al pote", ha promovido la clasifcación de los residuos orgánicos e inorgánicos al aplicar la regla de las tres R (Reducir, Reutilizar, Reciclar), y usa como herramientas la comunicación y la educación, para repercutir, de esta manera, en comportamientos individuales que tienen como base la cultura, la política social, y lo económico.

Durante este proceso, el profesional de enfermería ha sido un integrante importante del equipo de trabajo por su participación en la vinculación de la salud con el desarrollo social, por la responsabilidad directa e indirecta en la promoción de la salud, prevención y control de las enfermedades, así como en la conservación ambiental mediante la integración de sus conocimientos en educación e investigación para ejercer una labor integral de entrega y servicio, al fomentar un nivel óptimo de salud en las personas, las familias y la comunidad, y hacer de éste un trabajo importante para su desarrollo y reconocimiento en la sociedad. (Arango, 2009)

La estrategia de intervención para la adopción de hábitos y estilos de vida saludable es un reto para el quehacer del profesional de enfermería que labora en el área de salud pública quien trabaja en forma directa con la comunidad, por esto es un aprendiza- 
je elaborado desde la realidad local, a partir de la mirada crítica sobre lo que se hace y se deja de hacer por los allí implicados, al buscar modelos que partan de la realidad y contribuyan a la solución de los problemas de salud que más los afectan. El proceso se centra en el desarrollo de un sistema educativo conocido como Aprendizaje Basado en Problemas (ABP) (Morales, 2004) Barroows (1986) defne el ABP como un método de aprendizaje basado en el principio de usar problemas como punto de partida para la integración de los nuevos conocimientos. Por su parte, (Escribano y Del Valle, 2008) afrman que el $\mathrm{ABP}$ es un sistema didáctico que requiere que los estudiantes - para efectos de este proyecto, "los sujetos" - se involucren de forma activa en su propio aprendizaje hasta el punto de defnir un escenario de formación auto dirigida.

Esto justifca el hecho de que el trabajo con la comunidad se proyecte hacia la búsqueda de un cambio social necesario para la construcción de ambientes saludables de promoción y prevención que disminuyan los niveles de morbi- mortalidad existente (Escribano, 2008)

Las premisas, orientadoras de la teoría, la investigación y la aplicación en la práctica del Modelo Transteórico indican que (Cabrera, 2000)

- Ninguna teoría o modelo responde totalmente por la complejidad explicativa del cambio comportamental.

- El cambio es un proceso que se presenta como una secuencia de etapas.

- Las etapas son estables pero abiertas al cambio, tal como ocurre con los factores de riesgo comportamental.

- La mayoría de las poblaciones de riesgo no están preparadas para actuar y, por eso, no se benefcian de los tradiciona- les programas informativos, educativos o preventivos orientados a los riesgos comportamentales.

- Sin intervenciones planeadas según las etapas de cambio, las poblaciones en general y los grupos e individuos en particular, permanecerán detenidos en las etapas iniciales de cambio, sin motivación, ni intención de participar en las intervenciones o programas ofrecidos.

- La educación, la promoción y la prevención de la salud, pueden experimentar impactos nunca antes registrados si incorporasen un paradigma de etapas, distinto al ya clásico y superado de acción.

- Procesos y principios específcos de cambio necesitan ser aplicados a etapas de cambio específcas; las intervenciones deben diseñarse según las etapas de cambio propias de cada grupo o individuo.

- Los comportamientos están dominados de alguna manera por factores biológicos, sociales y de autocontrol.

- Las intervenciones requieren evolucionar de un reclutamiento reactivo de participantes a uno proactivo.

- El reto es avanzar hacia programas e intervenciones de la salud comportamental, de base poblacional, con apoyo de estrategias interactivas así como estrategias que den soporte ambiental a los cambios esperados.

\section{Variables del Modelo Transteórico}

El Modelo Transteórico explica el cambio espontáneo o inducido del comportamiento de los individuos según cinco variables o constructos teóricos intervinientes: etapas y procesos de cambio, balance decisorio, tentación y auto-efcacia. 


\section{Diagrama 1. Variables del Modelo Transteórico}

\begin{tabular}{|c|c|c|c|}
\hline \multirow{2}{*}{$\begin{array}{l}\text { ETAPAS DE CAMBIO } \\
\text { Precontemplación } \\
\text { Preparación } \\
\text { Acción } \\
\text { Mantenimiento }\end{array}$} & & \multicolumn{2}{|c|}{ PROCESO DE CAMBIO } \\
\hline & & $\begin{array}{l}\text { Concientización } \\
\text { Catarsis } \\
\text { Autoafrmación } \\
\text { Acondicionamiento } \\
\text { Relaciones de apoyo }\end{array}$ & $\begin{array}{l}\text { Autoevaluación } \\
\text { Revaluación ambiental } \\
\text { Liberación social } \\
\text { Control de estímulos } \\
\text { Adminsitración de } \\
\text { eventos }\end{array}$ \\
\hline \multirow{2}{*}{$\begin{array}{l}\text { BALANCE DECISORIO } \\
\text { Pros y contras para } \\
\text { cambiar }\end{array}$} & $\begin{array}{c}\text { COMPORTAMIENTO } \\
\text { OBJETIVO }\end{array}$ & \multicolumn{2}{|c|}{ TENTACIÓN } \\
\hline & & \multirow{2}{*}{\multicolumn{2}{|c|}{$\begin{array}{c}\text { Situaciones emocionales negativas } \\
\text { Situaciones emocionales positivas } \\
\text { Impulsos incontrolables }\end{array}$}} \\
\hline AUTOEFICACIA & & & \\
\hline
\end{tabular}

Fuente: PROCHASKA J, VELICER W. The transtheoretical model of health behavior chance. American Journal of Health Promotion 1997.

El Modelo Transteórico usa una dimensión temporal, las etapas del cambio, para integrar los procesos y principios explicativos del cambio comportamental de diferentes teorías. El cambio implica un fenómeno que ocurre con relación al tiempo, pero sorprendentemente casi ninguna teoría o modelo incluye un constructo representativo del tiempo.

El primer estadio de cambio es el de precontemplación, que es el estadio en el cual no hay intención para el cambio de conducta o deseo de adoptar hábitos nuevos. Para esta etapa se inicia el proceso de intervención y se utiliza como elementos básicos, preguntar al individuo la razón por la que no hace clasifcación de residuos en orgánicos e inorgánicos o si conoce la importancia de ello para él, su familia y el planeta. También, se propone proveer información acerca de la importancia y benefcios para la comunidad de lograr la clasifcación de los residuos sólidos. El objeto de este estadio es el de facilitarle al individuo el espacio para que piense en los riesgos ambientales y de los benefcios de un ambiente sano no contaminado. Para ello se mostraron videos con los cambios climáticos a causa de la acumulación de residuos indiscriminadamente. Además se desarrollaron talleres que permitían la elaboración de elementos decorativos y útiles fabricados a partir de residuos inorgánicos, de esta forma se evidencia cómo el reciclaje puede ser un medio de subsistencia para algunas personas, al igual que la elaboración de abono a partir de los residuos orgánicos como una posibilidad de incrementar el ingreso al convertirlo en empresa. En este estadio es indispensable que las personas retomen el gusto por las actividades, a través de las manualidades.

Un segundo estadio es el de contemplación, en el cual el sujeto piensa en la posibilidad de clasifcar los residuos en su casa en un futuro cercano. Una vez clasifcado en este estadio, se hace necesario capacitar- 
lo en la forma de realizar el proceso, con el objeto de que pueda avanzar a otro nivel o estadio. Para tal efecto debe quedar claro el conocimiento brindado sobre el tema y las oportunidades para que pueda involucrarse en actividades que correspondan en su nivel de destreza. Además, se debe seguir con las actividades de la fase anterior y con la enseñanza de nuevas manualidades que les permita ver mayor utilidad al proceso que realizan.

En este punto del modelo, se plantea que si la persona interioriza las estrategias anteriores estaría en el estadio de decisión. Decidiría, se prepararía o comenzaría a realizar pequeños cambios. Este es un punto crítico en los estadios de cambio, por ello, requiere de un aumento o eliminación de las barreras que impiden que la persona cambie. Además, se necesita que el docente le ayude a proponerse metas realistas. La estrategia conlleva la sustitución de conductas pasivas por otras que conduzcan a activarse. Punto clave es el estímulo constante, basado en los logros y no en aspectos generales (Cardinal, 1998).

Algunos autores como, Marcus (1992), sostienen que además de los estadios anteriores, es necesario incluir una fase de acción, que es cuando el individuo modifca su conducta, experiencias y ambiente para iniciar nuevos patrones de clasifcación y reciclaje. El periodo de inicio y los seis meses siguientes se consideran críticos, ya que es en este momento cuando la persona es más propensa a abandonar las nuevas conductas (Dishman, 1988). El cambio realizado en sus patrones, requiere de acompañamiento y refuerzos permanentes, por medio de las visitas domiciliarias de seguimiento donde se enfatiza en los logros y no en comentarios generales de éxito. Además, en este punto se facilita mucho la permanencia del sujeto en la etapa, si se desarrolla una cadena de apoyo como, por ejemplo, la familia, los niños escolares que actúan como multiplicadores, así es como los padres podrían involucrarse con sus hijos durante la semana en la actividad de clasifcación y reciclaje, que no sólo dé soporte a los cambios en la familia sino al cambio del entorno.

La última etapa de este modelo es la de mantenimiento, que es cuando el individuo lleva más de seis meses con los cambios de conducta. Un sujeto que se encuentra en este estadio, se le debe incentivar para que continúe en el proceso. En este momento, surge la necesidad de focalizar obstáculos, que impidan o minimicen las posibilidades de éxito (Cardinal, 1998).

\section{Metodología}

La investigación - acción, se desarrolló en cuatro barrios de la Comuna 9 de Bucaramanga correspondientes a San Martín, Torres de Alejandría, La Libertad y Diamante I, mediante la aplicación del Ciclo Cibernético de Transformación (CCT) (De Gregori, 2000), con fases de indagación-diagnóstico, planeación-programación y acción-evaluación, como una espiral autorrefexiva (Meza, 2010). A partir del problema detectado de la falta de clasifcación de los residuos sólidos, se implementó la estrategia educomunicativa, que consistía en enseñar la manera correcta de clasifcar, organizar, disponer parcialmente los residuos en el hogar en un recipiente con tapa, mientras el carro recolector se encarga de recibirlos para realizar su disposición fnal, reconocido un comportamiento de entrada y uno de salida después de la intervención. Se trabajó con una población aproximada de 800 habitantes y una muestra de 603 participantes principalmente amas de casa o quien hiciera las labores de la misma, para un porcentaje total de población intervenida de 75,2\% como población objeto. Para obtener multiplicadores se trabajó con los escolares de quinto y sexto grado de la institución educativa del barrio 
como población aliada, capacitándolos en el manejo y clasifcación de los residuos sólidos.

Es importante aclarar que se contó con el apoyo de la Policía Ambiental, de la Cooperativa de trabajo asociado reciclaje y servicios (Coopreser LTDA) y las empresas municipales de Aseo correspondientes a los barrios intervenidos (EMAB y Limpieza Urbana)

Las actividades educativas enfocadas hacia el manejo y clasifcación de los residuos sólidos se desarrollaron dos veces por semana. Para las amas de casa (población objeto) por medio de visita domiciliaria y taller grupal en el salón comunal, para los escolares (población aliada) en la institución educativa del barrio.

El eje de comunicación se trabajó mediante volantes, pasacalles, calcomanías y afches ubicados estratégicamente en los barrios y entregados en cada uno de los hogares objeto de intervención. Es este un proceso de ecoalfabetización que supuso comprender de qué modo están organizadas las cosas para sostener la red de la vida (Peacock, 2004)

\section{Discusión}

Prochaska y DiClemente (1985), proponen que la aplicación de la estrategia depende del estadio de cambio en que se encuentre el sujeto. Esto indica que la intervención debe tomar en cuenta lo anterior, para lograr aumentar la efectividad de la intervención. Por esta razón a continuación se muestra la conducta de entrada de la población objeto estudiada que se tuvo en cuenta para lograr enfatizar los procesos específcos durante el estadio concreto de cambio en que se encontraba el grupo intervenido.

\begin{tabular}{|c|c|c|}
\hline DETERMINACIÓN DE LA ETAPA & NÚMERO DE ENCUESTAS & PORCENTAJES \\
\hline PRECONTEMPLACIÓN & 14 & 2,3 \\
\hline CONTEMPLACIÓN & 96 & 16,1 \\
\hline DECISIÓN & 104 & 17,2 \\
\hline ACCIÓN & 250 & 41,4 \\
\hline MANTENIMIENTO & 139 & 23,0 \\
\hline TOTAL & $\mathbf{6 0 3}$ & $\mathbf{1 0 0 , 0}$ \\
\hline
\end{tabular}

Gráfca 1. Distribución porcentual según la determinacion de la etapa del comportamiento de los habitantes para separar los residuos

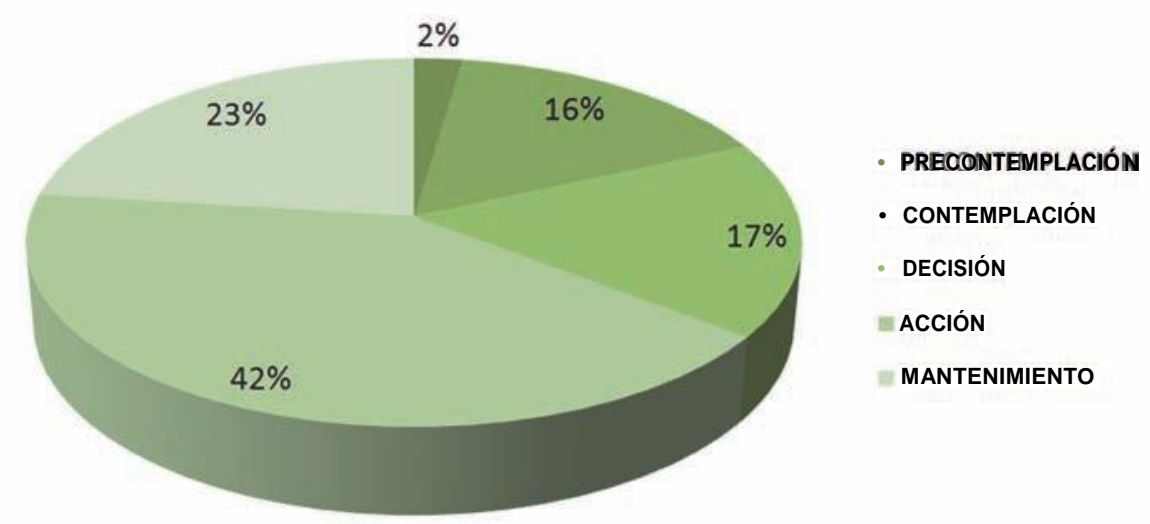


En consecuencia, uno de los puntos atractivos del modelo transteórico es que puede entenderse desde el inicio, cómo se encuentran los sujetos de estudio. Como en este caso donde se reconoce que el $2 \%$ de la población está en el estadio de precontemplación y un $16 \%$ en contemplación, grupo que requirió el mayor interés para implementar estrategias que propicien un cambio.

El $42 \%$ es el grupo que se encuentran en la etapa de acción y separa adecuadamente los residuos hace menos de 6 meses, grupo que posee sensibilización y concientización ambiental, necesaria para llevar a convertirlo en el estadio de mantenimiento y multiplicador para la comunidad. El objetivo del manejo integral de los residuos sólidos es proteger la salud de la población y mantener un ambiente agradable y sano. Esto se logrará si la comunidad maneja adecuadamente cada etapa, desde la producción y almacenamiento en el hogar hasta la disposición fnal de los residuos sólidos.

\begin{tabular}{|c|c|c|}
\hline DETERMINACIÓN DE LA ETAPA & NÚMERO DE ESCUESTAS & PORCENTAJES \\
\hline PRECONTEMPLACIÓN & 18 & 3,4 \\
\hline CONTEMPLACIÓN & 96 & 15,9 \\
\hline DECISIÓN & 56 & 9,1 \\
\hline ACCIÓN & 241 & 39,8 \\
\hline MANTENIMIENTO & 192 & 31,8 \\
\hline TOTAL & $\mathbf{6 0 3}$ & $\mathbf{1 0 0 , 0}$ \\
\hline
\end{tabular}

Gráfca 2 Rango porcentual de la determinación de la etapa de reciclaje en la que se encuentran las viviendas intervenidas

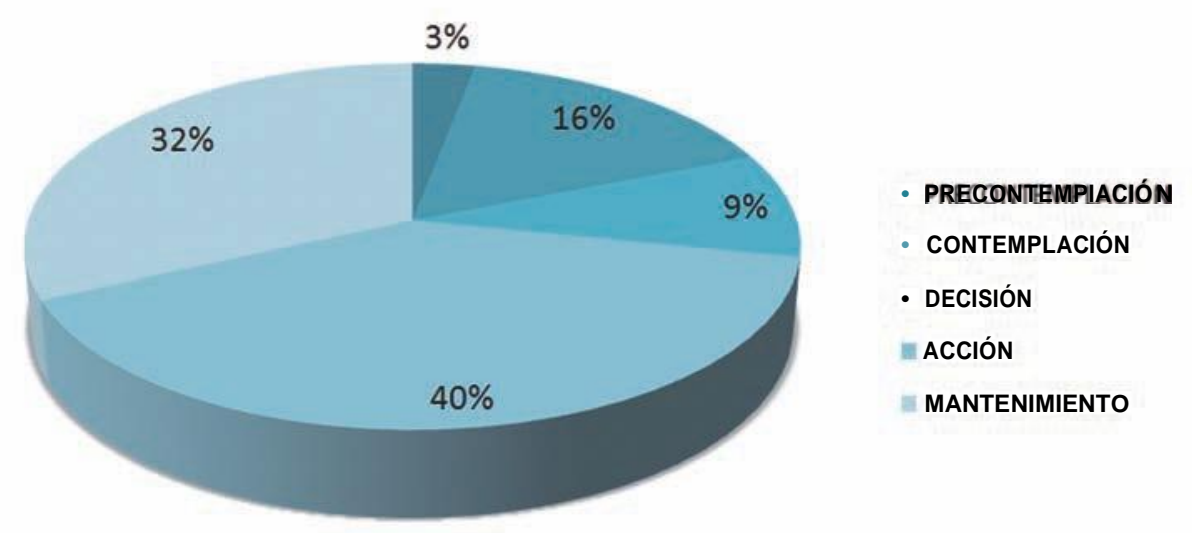

Fuente: los autores

El benefcio educativo y cultural al analizar estos resultados se manifesta en el cambio de actitud de muchas personas de la comunidad al realizar la separación de los residuos en la fuente. Después de la intervención realizada se encontró que un $40 \%$ de las amas de casa se clasifcaron en la etapa de acción, separa adecuadamente los residuos hace menos de 6 meses, $32 \%$ se encuentran en la etapa de mantenimiento separan adecuadamente los residuos hace más de 6 meses.

El benefcio ambiental se observa al recuperar recursos naturales renovables y no renovables; al separar los residuos sólidos 
reciclables como el papel, cartón, vidrio, plástico y metales para venderlos como materias primas en la elaboración de nuevos productos o transformándolos por medio de las manualidades en nuevos elementos para uso doméstico o decorativo. El inicio de la producción de abono orgánico resultado de los residuos vegetales es un valor agregado que permite la recuperación de suelos y fertilización de jardines. También se debe valorar el aumento de la vida útil del relleno sanitario del municipio, al disminuir la disposición fnal de residuos sólidos en la caja estacionaria con las actividades de reciclaje y la transformación de residuos vegetales en compost.

\section{Conclusión}

La implementación de la estrategia "Separe lo que bote antes de echarlo al pote" mediante el Modelo Transteórico con sus estadios de cambio, ha demostrado ser de gran impacto a la hora de estructurar intervenciones para cambiar conductas. Los fundamentos teóricos del modelo permiten valorar los estadios de cambio de forma sencilla además de desarrollar diferentes estrategias para movilizar a la persona de un estadio a otro, lo importante es el seguimiento y énfasis en el cambio conductual.

El potencial del modelo transteórico para asistir a los profesionales de enfermería, en la tarea de activar a la población en la campaña de educación ambiental para el manejo y clasifcación de residuos sólidos es signifcativo, especialmente cuando se aplican apropiadamente los procesos. Este dominio garantiza el uso pertinente de las estrategias que conducirán a movilizar a la persona a un nuevo estadio.

Con la participación activa de la población objeto como base de la estrategia y la población aliada como multiplicadores de la información; se busca generar un impacto en la sostenibilidad y preservación del medio ambiente, se logró contrarrestar las consecuencias ambientales, sociales y económicas que conlleva el inadecuado manejo y disposición de los residuos sólidos; al sensibilizar así de manera satisfactoria a las amas de casa y a estudiantes del grado tercero de los colegios aledaños a los barrios intervenidos sobre el manejo, clasifcación, disposición y reutilización de residuos sólidos por medio de actividades lúdico educativas.

\section{Referencias Bibliográfcas}

Alcaldía de Pereira. (2009) Caracterización de residuos sólidos urbanos en el municipio de Pereira.

Ángel, A. (2001). El Retorno de Ícaro. Cali: Corporación Universitaria Autónoma de Occidente.

Arango, María (et.al., 2009). Desarrollo de un Modelo de Extensión y Proyección Comunitaria de Intervenciones Efectivas en el Área de Salud Pública para la Adopción de Hábitos, Estilos de Vida Saludable y Sentido de Vida en la Población de la Comuna Nueve de Bucaramanga. Bucaramanga: Universidad Cooperativa de Colombia.

Barrows, H. (1996) Problem-Based learning in medicine and beyond: A brief overview.

Cardinal, B., Engels, K. and Zhu, W. (1998). Aplication of the transthearical model of behavior change to preadolescents physical activity and excercise behavior. Pedictric Excercise Sciense, 10.

Cabrera A., Gustavo. (2000). El Modelo Transteórico del Comportamiento en Salud. Revista de la Facultad Nacional de Salud Publica. Vol. 18 N. 002. Universidad de Antioquia, Medellín 2000.

Comisión Regional del Medio Ambiente. Región Metropolitana, 2006. Estudio caracterización de residuos sólidos domiciliarios en la región Metropolitana. Disponible en: http:/ / www.sinia.cl/1292/ articles-39508_pdf_informeF.pdf. Recuperado el 10 de agosto de 2010.

Dishmann, R. (1988) Excercise adherence. Champain, IL: Human Kinetics.

Escobar, M. (et al. 2010). Estrategias, métodos y técnicas efcaces en investigaciones para intervención y transformación de estilos de vida saludable mediante un pro- 
ceso de sistematización en la comuna número nueve de Bucaramanga.

Hamilton, E. (1981). La estrategia y la metodología de la evaluación de programas de desarrollo social" en Planifcación social en América Latina y el Caribe. Santiago de Chile.: ILPES/UNICEF.

Medicine and beyond: A brief overview. San Francisco: Education: Theory and Practice.

Morales, (2008) Aprendizaje Basado en Problemas. Madrid: Marcea

Prochaska. J, (1997). The transtheoretical model of health behavior chance. American Journal of Health Promotion. Restrepo, F. (2003). Manejo de residuos sólidos en el suroeste antioqueño. [Memorias en CD - ROM]. Medellín: Universidad Nacional.

Universidad Cooperativa De Colombia (2002). Programa de Enfermería. Caracterización del Escenario Socio - Cultural de la Comuna Nueve de la Ciudad de Bucaramanga. 
